OPEN ACCESS

Edited by:

Raffaele Zarrilli,

Università degli Studi di Napoli

Federico II, Italy

Reviewed by:

Maria Bagattini,

Università degli Studi di Napoli

Federico II, Italy

Xiaoting Hua,

Zhejiang University, China

Rita Berisio,

Consiglio Nazionale Delle Ricerche

(CNR), Italy

*Correspondence:

Gian Maria Rossolini

gianmaria.rossolini@unifi.it

Specialty section

This article was submitted to Antimicrobials, Resistance and

Chemotherapy,

a section of the journal

Frontiers in Microbiology

Received: 15 May 2018

Accepted: 18 July 2018

Published: 07 August 2018

Citation:

Cannatelli A, Principato S, Colavecchio OL, Pallecchi L and

Rossolini GM (2018) Synergistic Activity of Colistin in Combination With Resveratrol Against Colistin-Resistant

Gram-Negative Pathogens.

Front. Microbiol. 9:1808

doi: 10.3389/fmicb.2018.01808

\section{Synergistic Activity of Colistin in Combination With Resveratrol Against Colistin-Resistant Gram-Negative Pathogens}

\author{
Antonio Cannatelli ${ }^{1}$, Silvia Principato ${ }^{1}$, Olga L. Colavecchio ${ }^{2}$, Lucia Pallecchi ${ }^{1}$ and \\ Gian Maria Rossolini ${ }^{3,4 *}$
}

\begin{abstract}
${ }^{1}$ Department of Medical Biotechnologies, University of Siena, Siena, Italy, ${ }^{2}$ SOD Genetic Diagnostic, Florence Careggi University Hospital, Florence, Italy, ${ }^{3}$ Department of Experimental and Clinical Medicine, University of Florence, Florence, Italy, ${ }^{4}$ Clinical Microbiology and Virology Unit, Florence Careggi University Hospital, Florence, Italy
\end{abstract}

Objectives: In this study, we investigated the antimicrobial activity of resveratrol in combination with colistin, a last-resort agent for the treatment of severe infections caused by multidrug resistant Gram-negative pathogens.

Methods: The synergistic activity and the bactericidal activity of colistin in combination with resveratrol was investigated by checkerboard assays and time-kill assays, respectively. A total of 21 strains were investigated, including 16 strains of different species (Klebsiella pneumoniae, $n=6$, Escherichia coli, $n=6$; Citrobacter braakii, $n=1$; Stenotrophomonas malthophilia, $n=1$; Enterobacter cloaceae, $n=1$; Acinetobacter baumannii, $n=1$ ) with acquired colistin resistance, three colistin-susceptible $K$. pneumoniae precursors, and two strains of intrinsically colistin-resistant species (Serratia marcescens, $n=1$; Proteus mirabilis, $n=1$ ). Mechanisms of acquired colistin resistance included chromosomal mutations (i.e., $m g r B, p m r A B$ ) and plasmid genes (mcr-1, mcr-1.2).

Results: Resveratrol did not show any significant intrinsic antimicrobial activity. Overall, a relevant synergistic antimicrobial activity of resveratrol in combination with colistin was observed with all tested strains, except for the three colistin-susceptible $K$. pneumoniae strains, and for two mcr-1-positive E. coli strains. In time-kill assays, performed with 15 selected strains, the combination of colistin $2 \mathrm{mg} / \mathrm{L}$ plus resveratrol $128 \mathrm{mg} / \mathrm{L}$ was bactericidal with 11 strains, and bacteriostatic for the remaining ones.

Conclusions: Resveratrol was found to potentiate colistin activity against a wide panel of colistin-resistant strains, regardless of species and resistance mechanisms, which would deserve further investigation for potential clinical applications.

Keywords: colistin, resveratrol, colistin resistance, antibiotic resistance breakers, combination therapy 


\section{INTRODUCTION}

Resveratrol (3,5,4'-trihydroxy-trans-stilbene) is a stilbenoid compound found in numerous plants. Resveratrol has been investigated for potential therapeutic effects in various diseases (Chen et al., 2005; Albani et al., 2010; Sawda et al., 2010; Sun et al., 2010; Singh et al., 2014; Li et al., 2016, 2017) and has also shown the potential for antiviral (Abba et al., 2015; Lin et al., 2017a,b) and antibacterial activity against some pathogens, including Helicobacter pylori, Propionibacterium acnes, and Staphylococcus aureus (Mahady et al., 2003; Su et al., 2014; Taylor et al., 2014). Anti-oxydant activity and interaction with various molecular targets, including kinases, sirtuins, and cytokines, have been suggested as mechanisms responsible for resveratrol activity, although knowledge on this aspect remains limited (Kuršvietiene et al., 2016).

Polymyxins are old antibiotics that, until recently, were rarely used in the clinical setting except for the practice of Selective Digestive Decontamination (SDD), carried out in some ICU settings to reduce infections caused by microorganisms from oropharyngeal and gastrointestinal tracts (Abis et al., 2013; Bar-Yoseph et al., 2016; Rawson et al., 2016). Recently, due to the emergence of extremely drug resistant (XDR) strains of Gram-negative pathogens, such as carbapenemresistant Enterobacteriaceae (CRE) and carbapenem-resistant Acinetobacter sp. (CRA), polymyxins have regained a major role as last-resort agents for these infections, and their consumption has remarkably increased (Falagas and Kasiakou, 2005; Kaye et al., 2016). Unfortunately, also polymyxin resistance has emerged and is now increasingly reported, especially among CRE and CRA (Cannatelli et al., 2013; Monaco et al., 2014; Granata and Petrosillo, 2017; Jeannot et al., 2017; Nowak et al., 2017), further narrowing the treatment options.

In this study, we have tested the in vitro activity of resveratrol, alone and in combination with colistin, against a collection of colistin-resistant (COL-R) Gram-negative pathogens of different species. Despite the lack of any significant intrinsic antimicrobial activity, resveratrol exhibited a strong synergistic effect with colistin against many COL-R strains of different species, including Escherichia coli, Klebsiella pneumoniae, Enterobacter cloacae, Stenotrophomonas maltophilia, Citrobacter braakii, and also enterobacterial species that are naturally resistant to polymyxins (e.g., Proteus mirabilis and Serratia marcescens).

\section{MATERIALS AND METHODS}

\section{Bacterial Strains}

Bacterial strains investigated in this work are listed in Table $\mathbf{1 .}$ These included 18 COL- $\mathrm{R}$ strains of different species (A. baumannii, K. pneumoniae, E. coli, E. cloacae, S. maltophilia, C. braakii, P. mirabilis, and S. marcescens) and three colistinsusceptible (COL-S) K. pneumoniae that were precursors of three COL-R strains. For S. maltophilia, for which clinical breakpoints for colistin are not available, the definition as COL-R was arbitrarily based on the high-level colistin MIC (i.e., $32 \mathrm{mg} / \mathrm{L}$ ) as compared with the colistin MIC distribution for the species
(Sergio et al., 2017). For some strains, the mechanism of colistin resistance had been previously characterized (Table 1).

\section{Chemicals}

Colistin sulfate and resveratrol were obtained from SigmaAldrich (Saint Quentin Fallavier, France). Resveratrol (Thermo Fisher, Germany) was dissolved in dimethyl sulfoxide (DMSO) (Sigma-Aldrich, Saint Louis, USA) at a concentration of 20 $\mathrm{mg} / \mathrm{mL}$.

\section{In vitro Susceptibility Testing, Checkerboard Assays, and Time-Kill Assays}

MICs of colistin and resveratrol were determined by reference broth microdilution (Clinical and Laboratory Standards Institute, 2015) using cation-adjusted Mueller-Hinton broth (MHB) (bioMérieux, Florence, Italy). Colistin MICs were interpreted accordingly to the EUCAST clinical breakpoints, version 8.0 (www.eucast.org). Checkerboard assays to test the antimicrobial activity of combinations of colistin plus resveratrol were carried out as described previously (Tascini et al., 2013), using MHB and 96-well microtiter plates (Sarsted, Nümbrecht, Germany). Each well was inoculated with $50 \mu \mathrm{l}$ of a suspension of $5 \times 10^{5} \mathrm{CFU} / \mathrm{mL}$ of the test strain in a final volume of 100 $\mu l$. Inocula were prepared by direct suspension in MHB of bacteria grown overnight onto $\mathrm{MH}$ agar plates. Results were read after incubation at $35^{\circ} \mathrm{C}$ for $16-20 \mathrm{~h}$ and interpreted as follows: FICI $\leq 0.5$, synergism; FICI $>4.0$ antagonism; FICI $0.5-4$ no interaction. Data were obtained in at least two independent experiments.

Time-kill assays were performed in duplicate, by inoculating $5 \times 10^{6} \mathrm{CFU}$ of each strain into $2 \mathrm{~mL}$ of MHB in 24 Deep Well RB Block (Thermo Fisher Scientific, MA USA), at $35^{\circ} \mathrm{C}$, under static condition (Clinical and Laboratory Standards Institute, 2015). CFU counts were determined at different time points, by plating appropriate dilutions onto LB Agar (Sezonov et al., 2007).

In time kill assays, the DMSO concentration remained always below $1 \%(\mathrm{v} / \mathrm{v})$, as recommended by CLSI guidelines (Clinical and Laboratory Standards Institute, 2015). In MIC testing and checkerboard assays, the conditions with resveratrol concentrations of 256 and $512 \mathrm{mg} / \mathrm{L}$ contained DMSO concentrations higher than $1 \%$ (i.e., 1.36 and 2.72\%, respectively). Appropriate controls to exclude any potential synergistic activity between colistin and DMSO were always included.

\section{RESULTS AND DISCUSSION}

\section{Synergistic Activity of Colistin in Combination With Resveratrol in Checkerboard Assays}

A collection of 21 strains of eight different Gram-negative species were tested for susceptibility to resveratrol, colistin, and combinations thereof. The collection included 15 COL-R strains of species that are naturally susceptible to colistin (C. braakii, 


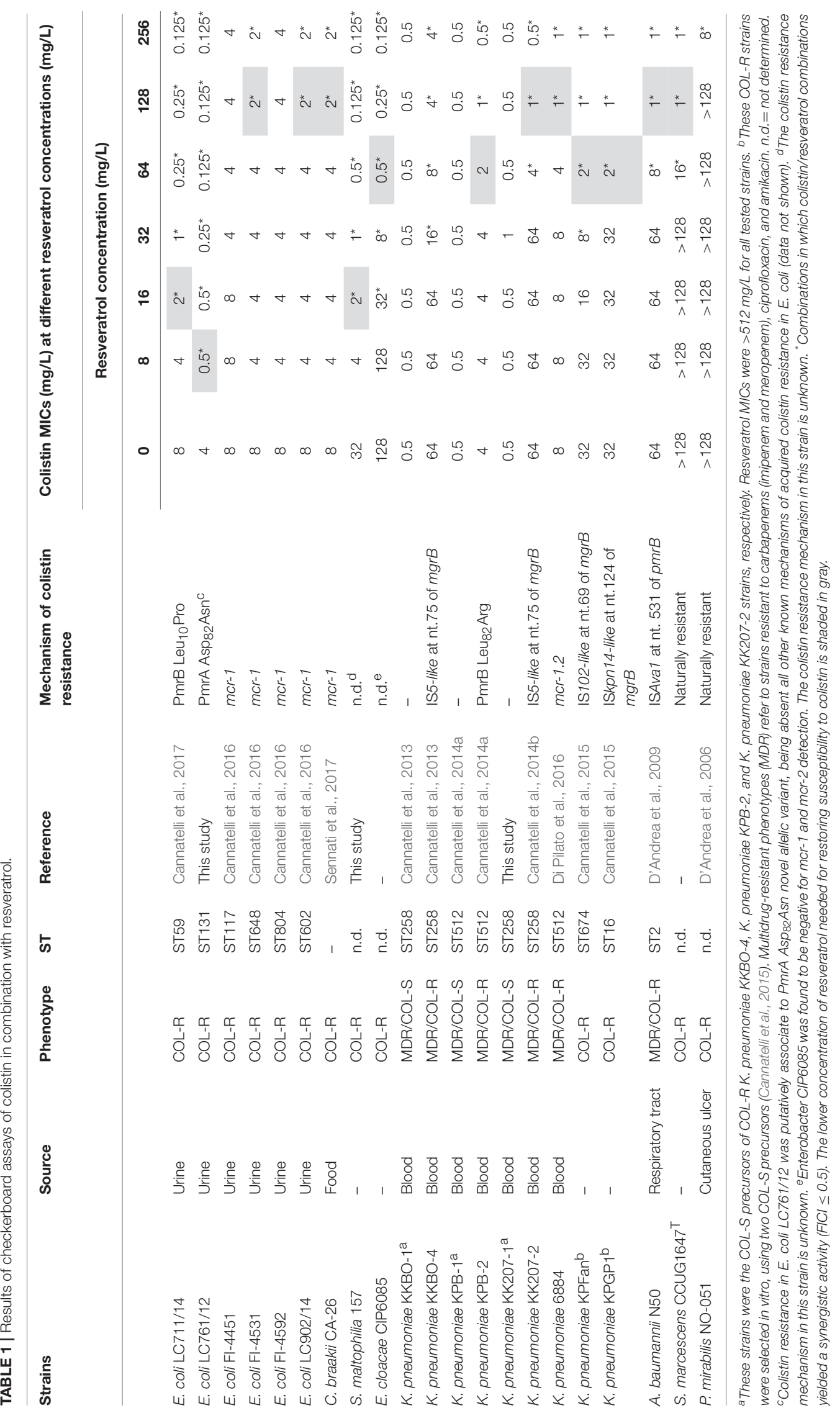


E. coli, K. pneumoniae, E. cloacae, A. baumannii), two COL$\mathrm{R}$ strains of naturally resistant species ( $S$. marcescens and $P$. mirabilis), and one S. maltophilia strain with high colistin MIC (that was considered COL-R for the purpose of this work). It also included three COL-S strains of K. pneumoniae, which were the precursors of three of the COL-R strains (Table 1).

MICs of resveratrol were $>512 \mathrm{mg} / \mathrm{L}$ for all tested strains, showing the lack of any intrinsic antimicrobial activity of resveratrol alone against these Gram-negative pathogens.

Checkerboard assays revealed a clear dose-dependent synergistic activity of resveratrol with colistin vs. COL-R strains of different species, including intrinsically resistant species such as $S$. marcescens and P. mirabilis. Synergism was not observed with two of the four COL-R E. coli strains carrying the mor-1 determinant and with the COL-S K. pneumoniae precursors of three COL-R strains (Table 1).

When synergism was evident, resveratrol was able to decrease colistin MICs to values equal or lower than the susceptibility breakpoint (i.e., $2 \mathrm{mg} / \mathrm{L}$ ) in most cases, at concentrations variable from 8 to $128 \mathrm{mg} / \mathrm{L}$. In particular, this was the case with four of the six COL-R E. coli, with five of the six COL-R $K$. pneumoniae, with the COL-R C. braakii, with the COL-R strains of A. baumanni, E. cloacae and S. maltophilia, and with the type strain of $S$. marcescens. The synergistic effect was observed in presence of different colistin resistance mechanisms and with strains of different clonal lineages, including representatives of known high risk clones (e.g., ST131 and ST59 for E. coli, or ST512 and ST258 for K. pneumoniae; Table 1).

\section{Synergistic Activity of Resveratrol and Colistin in Time-Kill Assays}

In order to investigate if colistin in combination with resveratrol had a bactericidal effect, time-kill assays were carried out with the 15 COL-R strains for which checkerboard assays had showed a synergistic effect. The colistin concentration used in timekill experiments corresponded to the clinical breakpoint for susceptibility $(2 \mathrm{mg} / \mathrm{L})$, or to $0.5 \times \mathrm{MIC}$ and $1 \times \mathrm{MIC}$, while the resveratrol concentration used was $128 \mathrm{mg} / \mathrm{L}$, which was able to inhibit the growth in most cases when combined with colistin at $2 \mathrm{mg} / \mathrm{L}$ (Table 1).

The time-kill assays showed a bactericidal activity (i.e., a reduction $\geq 3 \log _{10}$ of the initial bacterial inoculum) of resveratrol $128 \mathrm{mg} / \mathrm{L}$ in combination with colistin $2 \mathrm{mg} / \mathrm{L}$ with 11 of the 15 COL-R strains tested, while with four strains (E. coli FI-4531, K. pneumoniae KKBO-4, K. pneumoniae KK207-2, A. baumannii $\mathrm{N} 50)$ the combination exerted a static effect (Figure 1). Overall, with these strains when colistin was used at $0.5 \mathrm{X}$ MIC and $1 \mathrm{X}$ MIC in combination with resveratrol $128 \mathrm{mg} / \mathrm{L}$, a bactericidal

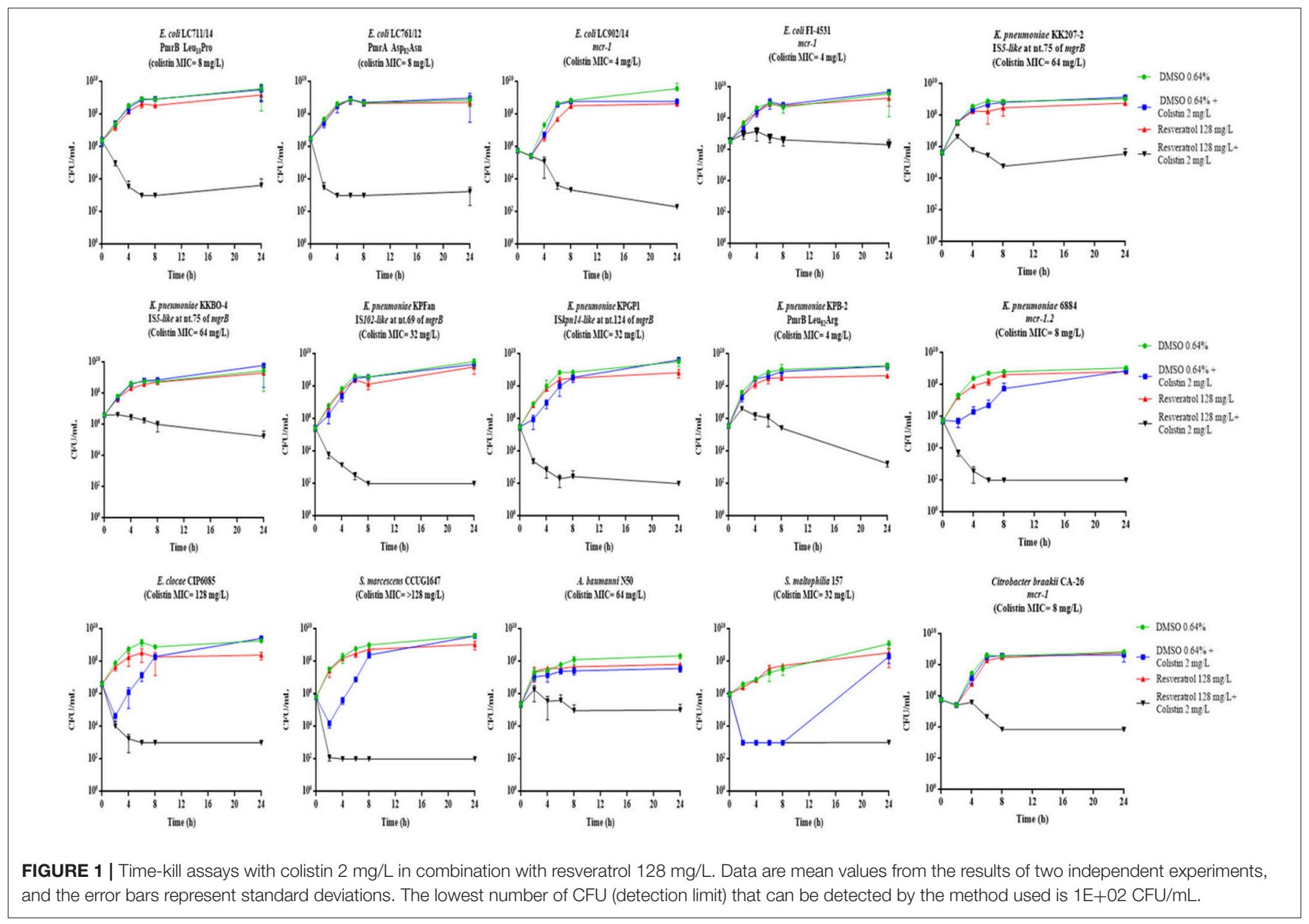


effect was observed, except for E. coli FI-4531, in which this combinations seem to be less effectives (Figure 2).

Altogether, the results were consistent with data obtained in checkerboard assays (Table 1). In fact, all strains exhibiting a colistin $\mathrm{MIC}<1 \mathrm{mg} / \mathrm{L}$ or $>2 \mathrm{mg} / \mathrm{L}$ in combination with resveratrol $128 \mathrm{mg} / \mathrm{L}$, showed a bactericidal or bacteriostatic effect in time-kill curves, respectively. On the other hand, a variable effect was observed with strains for which, in the presence of resveratrol $128 \mathrm{mg} / \mathrm{L}$, colistin MIC was lowered to 2 or $1 \mathrm{mg} / \mathrm{L}$ (Figure $\mathbf{1}$ ).

Considering the diversity of species tested, (C. braakii, E. coli, K. pneumoniae, E. cloacae, S. marcescens, A. baumannii, S. maltophilia) expressing different colistin resistance mechanisms, the "cidal/static" activity of colistin $2 \mathrm{mg} / \mathrm{L}$ in combination with resveratrol $128 \mathrm{mg} / \mathrm{L}$ did not appear to be dependent on species or the resistance mechanism.

The absence of any synergistic activity with COL-S strains could suggest a likely resveratrol interaction with the lipid A modification systems that are responsible for colistin resistance in COL-R strains. However, the mechanism of synergism observed between resveratrol and colistin with COL-R strains remains unknown and will be the subjects of further investigations.

\section{CONCLUDING REMARKS}

MDR and XDR Gram-negative bacteria (e.g., CRE and CRA) have been increasingly reported worldwide (Cannatelli et al., 2013; Monaco et al., 2014), and are listed among resistant pathogens with the highest priority for research and development of new antibiotics by the WHO (WHO, 2017).

Colistin remains one of the few antibiotics active against these pathogens, and represents a drug of last resort for the treatment of CRE and CRA severe infections (Falagas and Kasiakou, 2005; Kaye et al., 2016). It is also used for the Selective Digestive Decontamination (SDD) in combination with other agents (i.e., tobramycin, amphotericin B) (Abis et al., 2013; Rawson et al., 2016), and is increasingly administered for the management of chronic lung colonization by Pseudomonas aeruginosa in cystic fibrosis (Sherrard et al., 2014). In this perspective, the increasingly dissemination of colistin resistance in these pathogens represents a matter of public health concern (Cannatelli et al., 2013; Monaco et al., 2014; Granata and Petrosillo, 2017; Jeannot et al., 2017; Nowak et al., 2017).

This worrisome scenario has forced the scientific community to evaluate new therapeutic approaches to face the antibiotic resistance crisis. One promising strategy is offered by nonantibiotic drugs which overcome the resistance mechanism (Antibiotic Resistance Breakers; ARB) when combined with failing antibiotics (Brown, 2015). A well-proven example of such approach is represented by the new beta-lactamase inhibitors (i.e., avibactam, vaborbactam; Giani et al., 2016). Nonetheless, considering the multitude of resistance determinants and their rapid evolution potential, additional solutions must be implemented. Among the diverse approaches investigated over the last years, some natural compounds (i.e., resveratrol, quercetin, curcumin, pterostilbene), which have shown antibacterial properties (Su et al., 2014; Taylor et al., 2014; Hwang
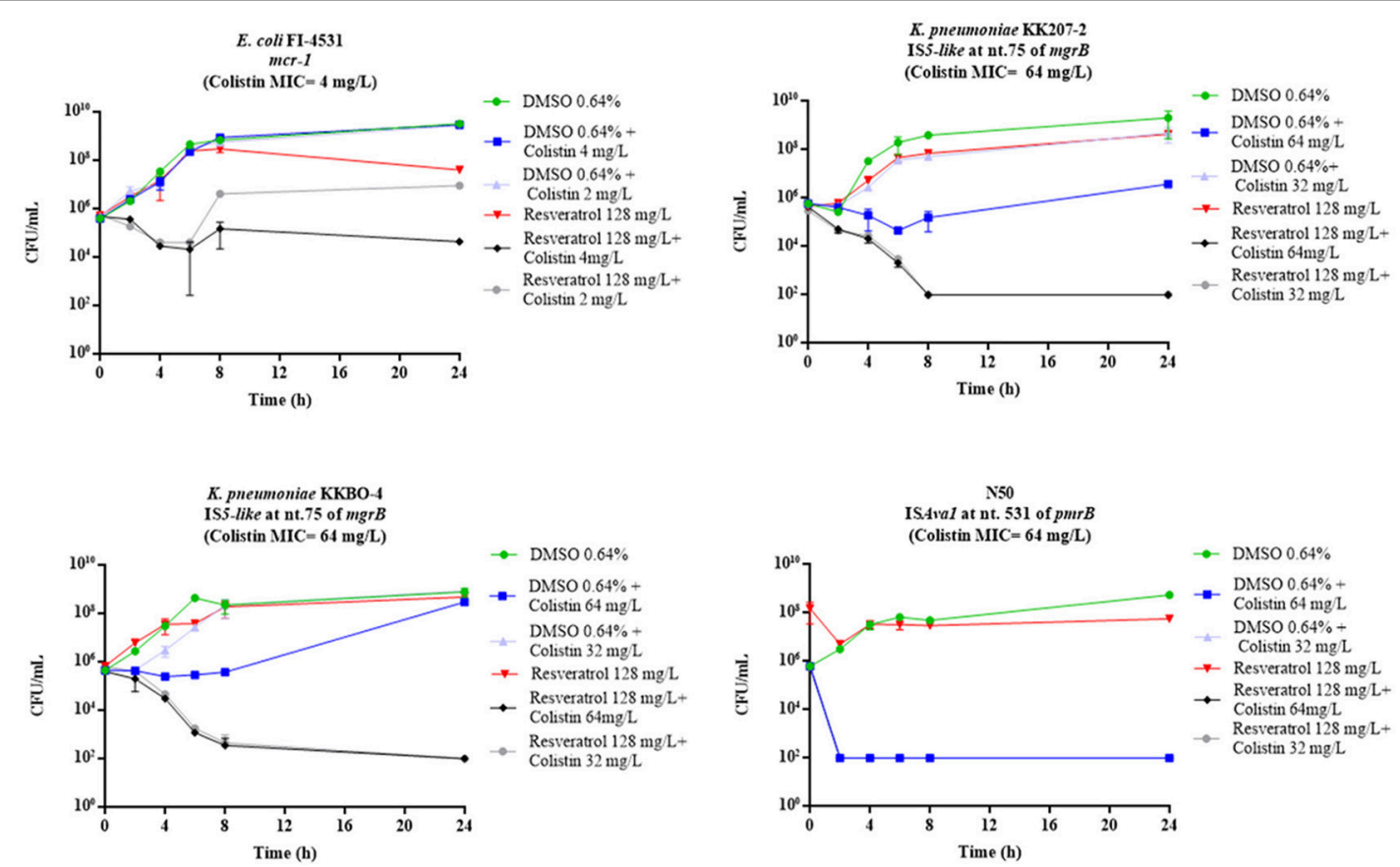

FIGURE 2 | Time-kill assays with colistin at 0.5 or 1 X MIC in combination with resveratrol $128 \mathrm{mg} / \mathrm{L}$, in the strains in which colistin $2 \mathrm{mg} / \mathrm{L}$ plus resveratrol $128 \mathrm{mg} / \mathrm{L}$ yielded a bacteriostatic effect. Data are mean values from the results of two independent experiments, and the error bars represent standard deviations. The lowest number of CFU (detection limit) that can be detected by the method used is $1 \mathrm{E}+02 \mathrm{CFU} / \mathrm{mL}$. 
and Lim, 2015; Kuršvietiene et al., 2016; Zhou et al., 2018), could also be of interest.

In this work we provided the first in vitro demonstration that resveratrol can act as an $\mathrm{ARB}$, potentiating colistin activity against a collection of COL-R Gram-negative pathogens, covering a wide panel of species and colistin resistance mechanisms. A limitation of this study was that the synergism between resveratrol and colistin was not tested with COL-R strains of $P$. aeruginosa, which at the time of the study were not available in our collection.

The potential to exploit this feature in vivo could depend on the resveratrol concentrations achievable in vivo, at different body sites. A number of pre-clinical and clinical studies have previously investigated resveratrol administered orally (ToméCarneiro et al., 2013), intravenously (Tomé-Carneiro et al., 2013), or by inhalation (Varricchio et al., 2014), but current knowledge on resveratrol pharmacokinetics in humans remain limited. When given orally, resveratrol is absorbed but readily metabolized, leading to a rather low bioavailability (Walle et al., 2004; Boocock et al., 2007; Tomé-Carneiro et al., 2013), while the non-absorbed fraction can be transformed by components of the gut microbiota (Tomé-Carneiro et al., 2013). Several resveratrol-derived metabolites have been identified in human and animals following oral or parenteral administration, including trans- and/or cis- forms of mono- and diglucuronides, mono- and disulfates, sulfoglucuronides, and dihydroresveratrol metabolities, which undergo renal and fecal excretion (Walle et al., 2004; Boocock et al., 2007; Tomé-Carneiro et al., 2013).

Consider that the activity of specific circulating resveratrol metabolites is still under debate, and the concentration of unchanged resveratrol in human urine, feaces and plasma has

\section{REFERENCES}

Abba, Y., Hassim, H., Hamzah, H., and Noordin, M. M. (2015). Antiviral activity of resveratrol against human and animal viruses. Adv. Virol. 2015:184241. doi: 10.1155/2015/184241

Abis, G. S., Stockmann, H. B., van Egmond, M., Bonjer, H. J., VandenbrouckeGrauls, C. M., and Oosterling, S. J. (2013). Selective decontamination of the digestive tract in gastrointestinal surgery: useful in infection prevention? A systematic review. J. Gastrointest. Surg. 17, 2172-2178. doi: 10.1007/s11605-013-2379-y

Albani, D., Polito, L., and Forloni, G. (2010). Sirtuins as novel targets for Alzheimer's disease and other neurodegenerative disorders: experimental and genetic evidence. J. Alzheimers Dis. 19, 11-26. doi: 10.3233/JAD-201 $0-1215$

Bar-Yoseph, H., Hussein, K., Braun, E., and Paul, M. (2016). Natural history and decolonization strategies for ESBL/carbapenem-resistant Enterobacteriaceae carriage: systematic review and meta-analysis. J. Antimicrob. Chemother. 71, 2729-2739. doi: 10.1093/jac/dkw221

Boocock, D. J., Faust, G. E., Patel, K. R., Schinas, A. M., Brown, V. A., Ducharme, M. P., et al. (2007). Phase I dose escalation pharmacokinetic study in healthy volunteers of resveratrol, a potential cancer chemopreventive agent. Cancer Epidemiol. Biomarkers Prev. 16, 1246-1252. doi: 10.1158/1055-9965.EPI-07-0022

Brown, D. (2015). Antibiotic resistance breakers: can repurposed drugs fill the antibiotic discovery void? Nat. Rev. Drug Discov. 14, 821-832. doi: $10.1038 / \operatorname{nrd} 4675$ still not been clearly determined, the use of colistin/resveratrol combinations for Selective Digestive Decontamination or treatment of urinary and systemic infections would deserve further investigation.

The most promising setting to exploit the synergism between resveratrol and colistin would be that of respiratory tract infections, where the administration of inhaled formulations of resveratrol might overcome the issues related to low bioavailability and metabolism of this compound. Being resveratrol also administered by nebulization in humans (Varricchio et al., 2014), the potential efficacy of colistin/resveratrol inhaled formulations could deserve further attention, especially in cases of chronic lung colonization by difficult-to-treat Gram-negatives, such as in cystic fibrosis, chronic obstructive pulmonary disease or bronchiectasis not related to cystic fibrosis.

Present results represent a proof of principle for further studies aimed at evaluating the potential role of resveratrol as colistin ARB in other in vitro (e.g., biofilm susceptibility testing) and in vivo models (e.g., Selective decontamination of the digestive tract).

- "The content is object of Italian Patent Application No. 102017000025738 filed on 08.03.2017"

- "The content is object of International Patent Application No. PCT/EP2018/055595 filed on 22.05.2018."

\section{AUTHOR CONTRIBUTIONS}

AC, LP and GR: study design, data analysis or interpretation, manuscript preparation. AC, SP, and OC: experimental studies, statistical analysis.
Cannatelli, A., D’Andrea, M. M., Giani, T., Di Pilato, V., Arena, F., Ambretti, S., et al. (2013). In vivo emergence of colistin resistance in Klebsiella pneumoniae producing KPC-type carbapenemases mediated by insertional inactivation of the PhoQ/PhoP mgrB regulator. Antimicrob. Agents Chemother. 57, 5521-5526. doi: 10.1128/AAC.01480-13

Cannatelli, A., Di Pilato, V., Giani, T., Arena, F., Ambretti, S., Gaibani, P., et al. (2014a). In vivo evolution to colistin resistance by PmrB sensor kinase mutation in KPC-producing Klebsiella pneumoniae is associated with lowdosage colistin treatment. Antimicrob. Agents Chemother. 58, 4399-4403. doi: 10.1128/AAC.02555-14

Cannatelli, A., Giani, T., Aiezza, N., Di Pilato, V., Principe, L., Luzzaro, F., et al. (2017). An allelic variant of the PmrB sensor kinase responsible for colistin resistance in an Escherichia coli strain of clinical origin. Sci. Rep. 7:5071. doi: 10.1038/s41598-017-05167-6

Cannatelli, A., Giani, T., Antonelli, A., Principe, L., Luzzaro, F., and Rossolini, G. M. (2016). First detection of the $m c r-1$ colistin resistance gene in Escherichia coli in Italy. Antimicrob. Agents Chemother. 60, 3257-3258. doi: 10.1128/AAC.00246-16

Cannatelli, A., Giani, T., D’Andrea, M. M., Di Pilato, V., Arena, F., Conte, V., et al. (2014b). MgrB inactivation is a common mechanism of colistin resistance in KPC-producing Klebsiella pneumoniae of clinical origin. Antimicrob. Agents Chemother. 58, 5696-5703. doi: 10.1128/AAC.03110-14

Cannatelli, A., Santos-Lopez, A., Giani, T., Gonzalez-Zorn, B., Rossolini, G. M., et al. (2015). Polymyxin resistance caused by $\operatorname{mgrB}$ inactivation is not associated with significant biological cost in Klebsiella pneumoniae. Antimicrob. Agents Chemother. 59, 2898-2900. doi: 10.1128/AAC.04998-14 
Chen, C. Y., Jang, J. H., Li, M. H., and Surh, Y., J. (2005). Resveratrol upregulates heme oxygenase-1 expression via activation of NF-E2-related factor 2 in PC12 cells. Biochem. Biophys Res. Commun. 331, 993-1000. doi: 10.1016/j.bbrc.2005.03.237

D’Andrea, M. M., Giani, T., D’Arezzo, S., Capone, A., Petrosillo, N., Visca, P., et al. (2009). Characterization of pABVA01, a plasmid encoding the OXA-24 carbapenemase from Italian isolates of Acinetobacter baumannii. Antimicrob. Agents Chemother. 53, 3528-3533. doi: 10.1128/AAC.00178-09

D’Andrea, M. M., Nucleo, E., Luzzaro, F., Giani, T., Migliavacca, R., Vailati, F., et al. (2006). CMY-16, a novel acquired AmpC-type beta-lactamase of the CMY/LAT lineage in multifocal monophyletic isolates of Proteus mirabilis from northern Italy. Antimicrob. Agents Chemother. 50, 618-624. doi: 10.1128/AAC.50.2.618-624.2006

Di Pilato, V., Arena, F., Tascini, C., Cannatelli,A., Henrici De Angelis, L., Fortunato, S., et al. (2016). $m c r-1.2$, a new mcr variant carried on a transferable plasmid from a colistin-resistant KPC carbapenemase-producing Klebsiella pneumoniae strain of sequence type 512. Antimicrob. Agents Chemother. 60, 5612-5615. doi: 10.1128/AAC.01075-16

Falagas, M. E., and Kasiakou, S. K. (2005). Colistin: the revival of polymyxins for the management of multidrug-resistant gram-negative bacterial infections. Clin. Infect. Dis. 40, 1333-1341. doi: 10.1086/429323

Giani, T., Cannatelli, A., Di Pilato, V., Testa, R., Nichols, W. W., and Rossolini, G. M. (2016). Inhibitory activity of avibactam against selected $\beta$-lactamases expressed in an isogenic Escherichia coli strain. Diagn. Microbiol. Infect. Dis. 86, 83-85. doi: 10.1016/j.diagmicrobio.2016.03.002

Granata, G., and Petrosillo, N. (2017). Resistance to colistin in Klebsiella pneumoniae: a 4.0 strain? Infect. Dis. Rep. 9:7104. doi: 10.4081/idr.2017.7104

Hwang, D., and Lim, Y. H. (2015). Resveratrol antibacterial activity against Escherichia coli is mediated by Z-ring formation inhibition via suppression of FtsZ expression. Sci. Rep. 5:10029. doi: 10.1038/srep10029

Jeannot, K., Bolard, A., and Plésiat, P. (2017). Resistance to polymyxins in gram-negative organisms. Int. J. Antimicrob. Agents. 49, 526-535. doi: 10.1016/j.ijantimicag.2016.11.029

Kaye, K. S., Pogue, J. M., Tran, T. B., Nation, R. L., and Li, J. (2016). Agents of last resort: polymyxin resistance. Infect. Dis. Clin. North Am. 30, 391-414. doi: 10.1016/j.idc.2016.02.005

Kuršvietiene, L., Stanevičiene, I., Mongirdiene, A., and Bernatoniene, J. (2016). Multiplicity of effects and health benefits of resveratrol. Medicina 52, 148-155. doi: 10.1016/j.medici.2016.03.003

Li, W., Cao, L., Chen, X., Lei, J., and Ma, Q. (2016). Resveratrol inhibits hypoxiadriven ROS-induced invasive and migratory ability of pancreatic cancer cells via suppression of the Hedgehog signaling pathway. Oncol. Rep. 35, 1718-1726. doi: 10.3892/or.2015.4504

Li, Y. R., Li, S., and Lin, C. C. (2017). Effect of resveratrol and pterostilbene on aging and longevity. Biofactors 44, 69-82. doi: 10.1002/biof.1400

Lin, S. C., Chen, M. C., Li, S., Lin, C. C., and Wang, T. T. (2017a). Antiviral activity of nobiletin against chikungunya virus in vitro. Antivir. Ther. 22, 689-697. doi: 10.3851/IMP3167

Lin, S. C., Ho, C. T., Chuo, W. H., Li, S., Wang, T. T., and Lin, C. C. (2017b). Effective inhibition of MERS-CoV infection by resveratrol. BMC Infect. Dis. 17:144. doi: 10.1186/s12879-017-2253-8

Mahady, G. B., Pendland, S. L., and Chadwick, L. R. (2003). Resveratrol and red wine extracts inhibit the growth of CagA + strains of Helicobacter pylori in vitro. Am. J. Gastroenterol. 98, 1440-1441. doi: 10.1111/j.1572-0241.2003.07513.x

Monaco, M., Giani, T., Raffone, M., Arena, F., Garcia-Fernandez, A., Pollini, S., et al. (2014). Colistin resistance superimposed to endemic carbapenem-resistant Klebsiella pneumoniae: a rapidly evolving problem in Italy, November 2013 to April 2014. Euro Surveill. 19:20939. doi: 10.2807/1560-7917.ES2014.19.42.20939

Nowak, J., Zander, E., Stefanik, D., Higgins, P. G., Roca, I., Vila, J., et al. (2017). High incidence of pandrug-resistant Acinetobacter baumannii isolates collected from patients with ventilator-associated pneumonia in Greece, Italy and Spain as part of the MagicBullet clinical trial. J. Antimicrob. Chemother. 72, 3277-3282. doi: 10.1093/jac/dkx322

Rawson, T. M., Moore, L. S., Hatcher, J. C., Donaldson, H., and Holmes, A. H. (2016). Plasmid-mediated colistin resistance mechanisms: is it time to revise our approach to selective digestive decontamination? Lancet Infect. Dis. 16, 149-150. doi: 10.1016/S1473-3099(15)00539-3
Sawda, C., Moussa, C., and Turner, R. S. (2010). Resveratrol for Alzheimer's disease. Ann. NY. Acad. Sci. 1403, 142-149. doi: 10.1111/nyas.13431

Sennati, S., Di Pilato, V., Riccobono, E., Di Maggio, T., Villagran, A. L., Pallecchi, L., et al. (2017). Citrobacter braakii carrying plasmid-borne mcr-1 colistin resistance gene from ready-to-eat food from a market in the Chaco region of Bolivia. J. Antimicrob. Chemother. 72, 2127-2129. doi: 10.1093/jac/dkx078

Sergio, F., Pallecchi, L., Landini, G., Di Maggio, T., Cariani, L., Blasi, F., et al. (2017). "Antimicrobial activity of N-acetylcysteine against Stenotrophomonas maltophilia and Burkholderia cepacia complex grown in planktonic phase and in biofilm," in Conference: ERS International Congress (Vienna).

Sezonov, G., Joseleau-Petit, D., and D'Ari, R. (2007). Escherichia coli physiology in Luria-Bertani broth. J. Bacteriol. 189, 8746-8749. doi: 10.1128/JB.01368-07

Sherrard, L. J., Tunney, M. M., and Elborn, J. S. (2014). Antimicrobial resistance in the respiratory microbiota of people with cystic fibrosis. Lancet 384, 703-713. doi: 10.1016/S0140-6736(14)61137-5

Singh, B., Shoulson, R., Chatterjee, A., Ronghe, A., Bhat, N. K., Dim, D. C., et al. (2014). Resveratrol inhibits estrogen-induced breast carcinogenesis through induction of NRF2-mediated protective pathways. Carcinogenesis 35, 1872-1880. doi: 10.1093/carcin/bgu120

Clinical and Laboratory Standards Institute, (2015). Methods for Dilution Antimicrobial Susceptibility Test for Bacteria that Grow Aerobically-10th Edn: Approved Standard M07-A10, NCCLS. Wayne, PA.

Su, Y., Ma, L., Wen, Y., Wang, H., and Zhang, S. (2014). Studies of the in vitro antibacterial activities of several polyphenols against clinical isolates of methicillin-resistant Staphylococcus aureus. Molecules 19, 12630-12639. doi: 10.3390/molecules 190812630

Sun, A. Y., Wang, Q., Simonyi, A., and Sun, G. Y. (2010). Resveratrol as a therapeutic agent for neurodegenerative diseases. Mol. Neurobiol. 41, 375-383. doi: 10.1007/s12035-010-8111-y

Tascini, C., Tagliaferri, E., Giani, T., Leonildi, A., Flammini, S., Casini, B., et al. (2013). Synergistic activity of colistin plus rifampin against colistin-resistant KPC-producing Klebsiella pneumoniae. Antimicrob. Agents Chemother. 57, 3990-3993. doi: 10.1128/AAC.00179-13

Taylor, E. J., Yu, Y., Champer, J., and Kim, J. (2014). Resveratrol demonstrates antimicrobial effects against Propionibacterium acnes in vitro. Dermatol. Ther. 4, 249-257. doi: 10.1007/s13555-014-0063-0

Tomé-Carneiro, J., Larrosa, M., González-Sarrías, A., Tomás-Barberán, F. A., García-Conesa, M. T., and Espín, J. C. (2013). Resveratrol and clinical trials: the crossroad from in vitro studies to human evidence. Curr. Pharm. Des. 19, 6064-6093. doi: 10.2174/13816128113199990407

Varricchio, A. M., Capasso, M., Della Volpe, A., Malafronte, L., Mansi, N., Varricchio, A., et al. (2014). Resveratrol plus carboxymethyl- $\beta$-glucan in children with recurrent respiratory infections: a preliminary and real-life experience. Ital. J. Pediatr. 40:93. doi: 10.1186/s13052-014-0093-3

Walle, T., Hsieh, F., DeLegge, M. H., Oatis, J. E. Jr., and Walle, U. K. (2004). High absorption but very low bioavailability of oral resveratrol in humans. Drug Metab. Dispos. 32, 1377-1382. doi: 10.1124/dmd.104.000885

WHO (2017) Essential Medicines and Health Products: Global Priority List of Antibiotic-Resistant Bacteria to Guide Research, Discovery, and Development of New Antibiotics. Available online at: http://www.who.int/medicines/ publications/global-priority-list-antibiotic-resistant-bacteria

Zhou, Y., Liu, S., Wang, T., Li, H., Tang, S., Wang, J., et al. (2018). Pterostilbene, a potential MCR-1 inhibitor that enhances the efficacy of polymyxin B. Antimicrob. Agents Chemother. 16:AAC.02146-17. doi: 10.1128/AAC.02146-17

Conflict of Interest Statement: The authors declare that the research was conducted in the absence of any commercial or financial relationships that could be construed as a potential conflict of interest.

The reviewer MB and handling Editor declared their shared affiliation.

Copyright (c) 2018 Cannatelli, Principato, Colavecchio, Pallecchi and Rossolini. This is an open-access article distributed under the terms of the Creative Commons Attribution License (CC BY). The use, distribution or reproduction in other forums is permitted, provided the original author(s) and the copyright owner(s) are credited and that the original publication in this journal is cited, in accordance with accepted academic practice. No use, distribution or reproduction is permitted which does not comply with these terms. 